\title{
The Role of Variational Principles of Mechanics \\ in the Study of the Evolution \\ of Non-Conservative Dynamical Systems
}

\author{
Evgeny A. Spirin, \\ Mikhail P. Golovin and Konstantin S. Fediy* \\ Siberian Federal University \\ 79 Svobodny, Krasnoyarsk, 660041, Russia
}

Received 15.08.2016, received in revised form 20.10.2016, accepted 24.11.2016

The article covers the basics of variational principles of mechanics applied to a material point is a brief historical background of variational methods mechanics, considered classical variational problem with fixed ends, shows the relationship of the law of conservation of energy to the principle of stationary action, as well as obtaining the characteristic function of a non-conservative system as an example of a mechanical system Linear friction. On the basis of Noether's theorem, shows the relationship of the first integral of the mechanical system, with its characteristic function, and interpretation made the first integral non-conservative mechanical system with linear friction.

Keywords: Lagrange function, dynamical systems, applied mathematics, calculus of variations, Hamilton's principle.

Citation: Spirin E.A., Golovin M.P., Fediy K.S. The role of variational principles of mechanics in the study of the evolution of non-conservative dynamical systems, J. Sib. Fed. Univ. Eng. technol., 2016, 9(7), 1068-1078. DOI: 10.17516/1999-494X-20169-7-1068-1078.

(c) Siberian Federal University. All rights reserved

* Corresponding author E-mail address: fediy_K@mail.ru 


\section{Роль вариационных принципов механики \\ в исследовании эволюции \\ неконсервативных динамических систем}

Е.А. Спирин, М.П. Головин, К.С. Федий

Сибирский федеральный университет

Россия, 660041, Красноярск, пр. Свободный, 79

В статье рассмотрены основы вариационных принципов механики применительно к материальной точке, приведена краткая историческая справка развития вариационных методов механики, рассмотрена классическая вариационная задача с закрепленными кониами, показана связь закона сохранения энергии с принципом стационарности действия, а также представлено получение характеристической функции неконсервативной системы на примере механической системы с линейным трением. На основе теоремы Нетер показана связь первого интеграла механической системы с ее характеристической функиией и выполнена интерпретация первого интеграла неконсервативной механической системь с линейным трением.

Ключевые слова: функция Лагранжа, динамическая система, прикладная математика, вариационное исчисление, принцип Гамильтона.

Принципами механики называются исходные положения, отражающие столь общие закономерности механических явлений, что из них как следствия можно получить все уравнения, определяющие движение механической системы. В ходе развития механики был установлен ряд таких принципов, каждый из которых может быть положен в основу механики. Эти принципы подразделяют на невариационные и вариационные.

Невариационные принципы механики непосредственно устанавливают закономерности движения, совершаемого системой под действием приложенных к ней сил. К этим принципам относятся, например, 2-й закон Ньютона, а также принцип Д’Аламбера.

Определение закона движения материальной точки является основной задачей теоретической механики, непосредственное интегрирование уравнений Ньютона не самый удобный способ ее решения.

Содержание вариационных принципов состоит в том, что они устанавливают свойства, позволяющие отличить истинное, т.е. фактически происходящее под действием заданных сил, движение механической системы от всевозможных её движений. Обычно эти свойства состоят в том, что для истинного движения некоторая физическая величина, зависящая от характеристик системы, имеет наименышее значение по сравнению с её значениями во всех рассматриваемых возможных движениях. При этом вариационные принципы могут отличаться друг от друга видом указанной физической величины и особенностями рассматриваемых возможных движений, а также особенностями самих механических систем, для которых эти принципы справедливы. Использование вариационных принципов требует применения методов вариационного исчисления.

Многие ведущие ученые внесли существенный вклад в развитие вариационных принципов аналитической механики [1]. 
Мопертюи (1698-1759) предложил универсальную гипотезу, согласно которой любые события в природе описываются при помощи определенной величины, называемой действием, принимающей минимальной значение на истинном пути движения. Однако Мопертюи не смог четко определить величину, которая должна быть минимизирована.

Эйлер (1707-1783) внес существенный вклад в развитие теоретической механики, свои работы в области вариационной механики он начал с изучения «изопериметрических» задач, для которых нашел решение в явном виде. Хотя Эйлер и не сформулировал четкого принципа наименьшего действия, что было впервые сделано Лагранжем, его применения этого принципа к механическим задачам, по сути дела, эквивалентны лагранжевой явной формулировке.

Лагранж (1736-1813). Если принцип виртуальных перемещений и принцип Даламбера позволили рассматривать механическую систему как нечто целое, не разбивая ее на изолированные частицы, то уравнения Лагранжа добавили еще одно важное свойство - инвариантность относительно произвольных преобразований координат. Лагранж создал инструмент решения механических задач при помощи чистых вычислений, без каких бы то ни было физических или геометрических соображений, при условии, что кинетическая и потенциальная энергии заданы в аналитической форме. Метод использования дополнительных условий при помощи неопределенных множителей - открытие Лагранжа, которое играет важную роль в теоретической механике.

Гамильтон (1805-1865). Уравнения Лагранжа были довольно сложными дифференциальными уравнениями второго порядка. Гамильтон сумел преобразовать их в систему дифференциальных уравнений первого порядка с удвоенным числом переменных, позиционные координаты и импульсы рассматривались при этом как независимые переменные. Это простейшая и наиболее удобная форма, к которой могут быть приведены уравнения вариационной задачи, получившей название «канонические уравнения», данное им Якоби. Гамильтон, преобразовав принцип Даламбера, впервые дал точную формулировку принципа наименьшего действия. Форма, в которой применяли этот принцип Эйлер и Лагранж, справедлива лишь для консервативных (склерономных) систем.

Принцип наименьшего действия (стационарности действия) является фундаментальной основой лагранжевой и гамильтоновой формулировок механики. Не все физические системы имеют уравнения движения, которые можно получить из этого принципа, однако все фундаментальные взаимодействия ему подчиняются, в связи с чем этот принцип признан одним из ключевых положений современной физики. Получаемые с его помощью уравнения движения имеют название уравнений Эйлера - Лагранжа.

При исследовании одномерного механического движения принцип стационарности действия записывается в форме функционала движения [2,3]:

$$
S[x(t)]=\int_{t_{0}}^{t_{1}} L(x(t), \dot{x}(t), t) d t \rightarrow \exp r
$$

где $\dot{x}(t)=\frac{d x(t)}{d t}$.

Наша задача - нахождение истинного закона движения $x=x^{0}(t)$, удовлетворяющего уравнению (1). Решением данной задачи является решение классической задачи функционального анализа о «траектории с закрепленными концами». 


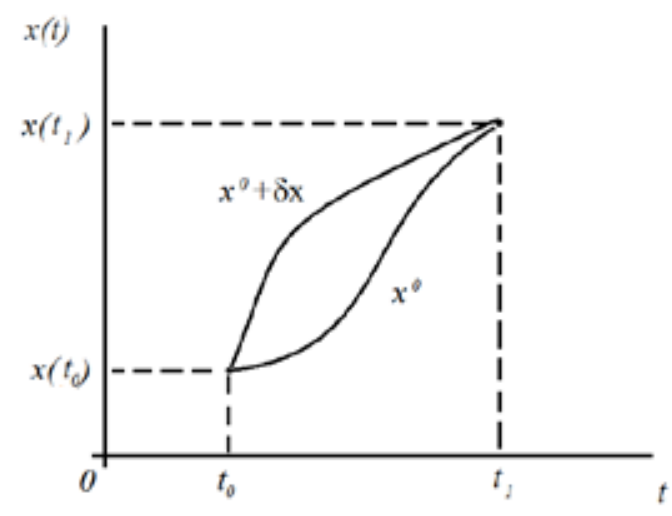

Рис. 1. Графическое представление траектории с закрепленными концами

Графически данную задачу можно представить в виде графика (рис. 1).

Из всего многообразия функций $x=x(t)$ истинным будет тот закон, который удовлетворяет условию (1) при движении из точки $x\left(t_{0}\right)$ в точку $x\left(t_{1}\right)$.

Любой неистинный закон будет отличаться от истинного закона на величину $\delta x=\delta x(t)$ и будет иметь вид

$$
x=x^{0}(t)+\delta x(t),
$$

где $\delta x(t)$ - произвольная функция.

Отклонение от $x^{0}(t)$ на величину $\delta x(t)$ будет приводить приращению функционала (1), которое составит

$$
\Delta S[x(t)]=\int_{t_{0}}^{t_{1}} L\left(x^{0}+\delta x, \dot{x}^{0}+\delta \dot{x}, t\right)-L\left(x^{0}, \dot{x}^{0}, t\right) d t .
$$

В уравнении (3) функция $\delta x(t)=x(t)-x^{0}(t)$ является вариацией аргумента функционала (1). На границах истинной траектории выполняется условие

$$
\begin{aligned}
& \delta x\left(t_{0}\right)=0, \\
& \delta x\left(t_{1}\right)=0 .
\end{aligned}
$$

Подынтегральная функция уравнения (3) может быть разложена по формуле Тейлора (5):

$$
\Delta L=d L+\frac{1}{2 !} d^{2} L+\cdots
$$

Линейная часть приращения функции $L$ описывается первым дифференциалом $d L$ :

$$
\frac{d L}{d t}=\frac{d}{d t}\left(L\left(x^{0}+\delta x, \dot{x}^{0}+\delta \dot{x}, t\right)-L\left(x^{0}, \dot{x}^{0}, t\right)\right)
$$

Определим производные:
a) $\frac{\partial L}{\partial t}=\frac{\partial L}{\partial x}\left(\frac{\partial x}{\partial t}+\frac{\delta x}{\partial t}\right)+\frac{\partial L}{\partial \dot{x}}\left(\frac{\partial \dot{x}}{\partial t}+\frac{\delta \dot{x}}{\partial t}\right)$ 
б) $\frac{\partial L}{\partial t}=\frac{\partial L}{\partial x}\left(\frac{\partial x}{\partial t}\right)+\frac{\partial L}{\partial \dot{x}}\left(\frac{\partial \dot{x}}{\partial t}\right)$

а-б) $\frac{\partial L}{\partial t}=\frac{\partial L}{\partial x} \frac{\delta x}{\partial t}+\frac{\partial L}{\partial \dot{x}} \frac{\delta \dot{x}}{\partial t}$.

С учетом (7) выражение (3) примет вид

$$
\Delta S[x(t)]=\int_{t_{0}}^{t_{t}}\left(\frac{\partial L}{\partial x} \delta x+\frac{\partial L}{\partial \dot{x}} \delta \dot{x}\right) d t
$$

Целью решения задач вариационного исчисления является поиск дифференциального уравнения, удовлетворяющего условию (1), для этого применяется основная лемма вариационного исчисления. При получении уравнения (8) лемма применяется при выносе за скобки общего множителя $\delta x$ и $\delta \dot{x}$.

Проинтегрировав по частям второе слагаемое уравнения (8), получим условие выполнения равенства (1):

$$
\Delta S[x(t)]=\int_{t_{0}}^{t_{1}}\left(\frac{\partial L}{\partial x}-\frac{d}{d t}\left(\frac{\partial L}{\partial \dot{x}}\right)\right) \delta x d t=0 .
$$

С учетом основной леммы вариационного исчисления условие (9) примет вид

$$
\frac{\partial L}{\partial x}-\frac{d}{d t}\left(\frac{\partial L}{\partial \dot{x}}\right)=0
$$

Выражение (10) называется уравнением Эйлера - Лагранжа. Уравнение (10) также можно получить, применив лемму Дюбуа - Реймона.

Рассматривая поведение функции $L$ относительно преобразований координат, можно получить ряд инвариантов механической системы. Одним из таких инвариантов является полная механическая энергия консервативной системы.

Полная производная функции $L(x(t), \dot{x}(t), t)$ по времени имеет вид (при условии, что она не зависит явно от времени)

$$
\frac{d L}{d t}=\frac{\partial L}{\partial x} \frac{\partial x}{\partial t}+\frac{\partial L}{\partial \dot{x}} \frac{\partial \dot{x}}{\partial t}
$$

С учетом (10) уравнение (11) примет вид

$$
\frac{d L}{d t}=\frac{d}{d t}\left(\frac{\partial L}{\partial \dot{x}}\right) \dot{x}+\frac{\partial L}{\partial \dot{x}} \ddot{x} .
$$

Уравнение (12) представляет собой производную произведения функций $\frac{\partial L}{\partial \dot{x}}$ и по времени, следовательно,

$$
\frac{d L}{d t}=\frac{d}{d t}\left(\frac{\partial L}{\partial \dot{x}}\right) \dot{x}+\frac{\partial L}{\partial \dot{x}} \ddot{x}=\frac{d}{d t}\left(\frac{\partial L}{\partial \dot{x}} \dot{x}\right)
$$

Уравнение (13) можно переписать в виде 


$$
\frac{d}{d t}\left(\frac{\partial L}{\partial \dot{x}} \dot{x}-L\right)=0
$$

Из уравнения (14) видно, что величина $E=\frac{\partial L}{\partial \dot{x}} \dot{x}-L$, называемая энергией, остается постоянной во времени.

Если ввести обозначения

$$
\begin{aligned}
& \frac{\partial L}{\partial \dot{x}}=p, \\
& \frac{\partial L}{\partial x}=f,
\end{aligned}
$$

где $p$ - обобщенный импульс; $f$ - обобщенная сила, то уравнение энергии примет вид

$$
E=p \dot{x}-L
$$

Закон движения материальной точки под действием сил, зависящих от пространственной координаты и её скорости, описанный с помощью уравнения Ньютона, имеет вид

$$
m \ddot{x}+n(x) \dot{x}=\varphi(x),
$$

где $m$ - масса материальной точки; $n(x)$ - коэффициент трения, зависящий от координаты; $\varphi(x)=-\frac{d \Pi}{d x}-$ возмущающая потенциальная сила.

Запишем уравнение Лагранжа второго рода для данной механической системы:

$$
\frac{d}{d t} \frac{\partial K}{\partial \dot{x}}-\frac{\partial K}{\partial x}=-\frac{\partial \Pi}{\partial x}-n(x) \frac{\partial K}{\partial \dot{x}}
$$

где $K=\frac{m \dot{x}^{2}}{2}$.

Умножим обе части уравнения (17) на неизвестную функцию $\mu$ и представим его в следующем виде:

$$
\mu \frac{d}{d t} \frac{\partial K}{\partial \dot{x}}+\mu \cdot n(x) \frac{\partial K}{\partial \dot{x}}=\frac{\partial}{\partial x}(\mu K-\mu \Pi) .
$$

Определим вид функции $\mu$ таким образом, чтобы левая часть уравнения (18) представляла собой производную по времени от произведения двух функций:

$$
\frac{d}{d t}\left(\mu \frac{\partial K}{\partial \dot{x}}\right)=\mu \frac{d}{d t} \frac{\partial K}{\partial \dot{x}}+\frac{d \mu}{d t} \frac{\partial K}{\partial \dot{x}}=\mu \frac{d}{d t} \frac{\partial K}{\partial \dot{x}}+\mu \cdot n(x) \frac{\partial K}{\partial \dot{x}}=\frac{d}{d t} \frac{\partial}{\partial \dot{x}}(\mu K-\mu \Pi) .
$$

Из уравнения (19) видно, что

$$
\frac{d \mu}{d t}=\mu \cdot n(x)
$$


Разделим обе части уравнения (22) на $\mu$ и, умножив на $d t \cdot \frac{\partial K^{-1}}{\partial \dot{x}}$, проинтегрируем:

$$
\int \frac{d \mu}{\mu}=\int n(x) d t=\ln (\mu)=n(x) t .
$$

Выразив из (21) $\mu$, получим

$$
\mu=e^{n(x) t} .
$$

С учетом уравнений (20), (21) и (24) имеет место равенство

$$
\frac{\partial}{\partial x}(\mu K-\mu \Pi)-\frac{d}{d t} \frac{\partial}{\partial \dot{x}}(\mu K-\mu \Pi)=0 .
$$

Уравнение (25) соответствует уравнению Эйлера (10). Таким образом, функция Лагранжа рассматриваемой механической системы имеет вид

$$
L=(K-\Pi) \mu .
$$

При этом действие такой системы описывается уравнением

$$
S[x(t)]=\int_{t_{0}}^{t_{1}}(K(\dot{x})-\Pi(x)) \mu(x, t) d t \rightarrow \text { extr } .
$$

Ковариантность уравнения (25) относительно замены координат и знание вида Лагранжиана (26) позволяет получать уравнения движения в удобной для решения форме, исследовать эволюцию динамических систем с линейным трением, а также определить преобразования симметрии и соответствующие им инварианты с применением теоремы Нётер.

Законы сохранения имеют глубокое происхождение, связанное с инвариантностью описания механической системы относительно некоторой группы преобразований времени и координат. Согласно теореме Нётер, каждой непрерывной симметрии физической системы соответствует некоторый закон сохранения. Симметрия - соответствие, неизменность (инвариантность), проявляемые при каких-либо изменениях, преобразованиях. Рассматривая различные преобразования координат, мы приходим к основным физическим симметриям [4].

При рассмотрении движения механической системы в пространстве могут быть использованы преобразования координат вида (28).

Таблица 1. Симметрия в физике

\begin{tabular}{|l|l|l|}
\hline \multicolumn{1}{|c|}{ Преобразование } & \multicolumn{1}{|c|}{$\begin{array}{c}\text { Соответствующая } \\
\text { инвариантность }\end{array}$} & \multicolumn{1}{|c|}{$\begin{array}{c}\text { Соответствующий } \\
\text { закон сохранения }\end{array}$} \\
\hline Трансляция времени & Однородность времени & энергии \\
\hline & Изотропность времени & четности \\
\hline Трансляция пространства & Однородность пространства & импульса \\
\hline Вращение пространства & Изотропность пространства & Момента импульса \\
\hline Группа Лоренца & Относительность Лоренц-инвариантность & 4-импульса \\
\hline Калибровочное преобразование & Калибровочная инвариантность & заряда \\
\hline
\end{tabular}




$$
\left\{\begin{array}{l}
t^{*}=f_{0}(t, x, a) \\
x_{i}^{*}=f_{i}(t, x, a)
\end{array}(i=1,2, \ldots, n),\right.
$$

где $a$ - независимый параметр.

Если рассматривать значения параметра $a$ достаточно близкими к нулевым, то преобразования (26) можно представить в виде

$$
\left\{\begin{array}{l}
t^{*}=f_{0}(t, x, \Delta a) \\
x_{i}^{*}=f_{i}(t, x, \Delta a)
\end{array}(i=1,2, \ldots, n) .\right.
$$

В результате разложения в ряд Тейлора преобразований (29), ограничиваясь членами, линейными относительно $\Delta a$, получают бесконечно малые преобразования, которые можно записать в виде

$$
\left\{\begin{array}{c}
t^{*}=t+\Delta t \\
x_{i}^{*}(t)=x_{i}(t)+\Delta x
\end{array}(i=1,2, \ldots, n) .\right.
$$

Например, возможные параллельные переносы трехмерной системы координат описываются преобразованием

$$
\left\{\begin{array}{c}
t^{*}=t \\
q_{i}^{*}=q_{i}+a_{i}
\end{array}(i=1,2,3) .\right.
$$

А бесконечно малое преобразование при этом имеет вид

$$
\left\{\begin{array}{c}
t^{*}=t \\
q_{i}^{*}=q_{i}+\varepsilon
\end{array}(i=1,2,3) .\right.
$$

Совокупность всех вращений декартовой системы координат на плоскости описывается преобразованием

$$
\left\{\begin{array}{c}
t^{*}=t \\
x^{*}=x \cdot \cos (a)-y \cdot \sin (a) \\
y^{*}=-x \cdot \sin (a)+y \cdot \cos (a)
\end{array} .\right.
$$

А его бесконечно малое преобразование таково:

$$
\left\{\begin{array}{c}
t^{*}=t \\
x^{*}=x-\varepsilon \cdot y . \\
y^{*}=y-\varepsilon \cdot x
\end{array}\right.
$$

Преобразования (30) называются преобразованиями симметрии, если выполняется условие инвариантности действия относительно этих преобразований. Данное условие имеет вид:

$$
\int_{t_{0}}^{t_{1}} L(x, \dot{x}, t) d t=\int_{t_{0}^{*}}^{t_{1}^{*}} L_{1}\left(x, \dot{x}, t^{*}\right) d t^{*},
$$

где функция $L_{1}-$ некоторая другая функция Лагранжа, позволяющая получить те же уравнения движения, что и $L$. 
Одним из следствий теоремы Нётер является уравнение первого интеграла движения, который с использованием функции Лагранжа описывается следующим уравнением:

$$
L \Delta t+\sum_{i=1}^{n} \frac{\partial L}{\partial \dot{x}} \delta x_{i}+\Delta \lambda=\Delta C
$$

где $\delta x_{i}=\Delta x-\dot{x} \Delta t$, a $\Delta \lambda-$ функция, определяемая из уравнения

$$
\frac{\partial L}{\partial t} \Delta t+\sum_{i=1}^{n} \frac{\partial L}{\partial x} \Delta x_{i}+\sum_{i=1}^{n} \frac{\partial L}{\partial \dot{x}} \Delta \dot{x}_{i}+L \frac{d}{d t} \Delta t=-\frac{d}{d t}(\Delta \lambda)
$$

Уравнение (37) применяется в качестве критерия для выяснения, является ли (28) преобразованием симметрии для исследуемой системы.

В качестве примера использования теоремы Нетер и функции Лагранжа на практике рассмотрим колебательную системы с силой трения, линейно зависящей от скорости и не зависящей от координаты. Лагранжиан такой системы с учётом (26) и (24) будет иметь вид

$$
L=(K-\Pi) \mu=\left(\frac{1}{2} m \dot{x}^{2}-\frac{1}{2} c x^{2}\right) e^{n t} .
$$

Обозначив

$$
2 \beta=n, k^{2}=c / m
$$

лагранжиан представим в виде

$$
L=\frac{1}{2}\left(\dot{x}^{2}-k^{2} x^{2}\right) e^{2 \beta t}
$$

Уравнение движения такой системы имеет вид

$$
\ddot{x}+2 \beta \dot{x}+k^{2} x=0 .
$$

Преобразованием симметрии такой системы будет преобразование вида

$$
\left\{\begin{array}{c}
t^{*}=t+\varepsilon \\
x^{*}=x-\varepsilon \beta x
\end{array}\right.
$$

Действительно, учитывая, что $\Delta t=\varepsilon, \Delta x=-\varepsilon \beta x, \Delta \dot{x}=-\varepsilon \beta \dot{x}$ из уравнения (37) получаем $\Delta \lambda=0$, а из уравнения (36) - первый интеграл системы:

$$
\left(\frac{1}{2} \dot{x}^{2}+\frac{1}{2} k^{2} x^{2}+\beta x \dot{x}\right) e^{2 \beta t}=C .
$$

Решение уравнения (39) можно представить в виде графика (рис. 2)

Для интерпретации физического смысла первого интеграла (43) рассмотрим во времени графики слагаемых первого множителя уравнения (43) и самого интеграла (рис. 3).

Таким образом, интеграл (41) играет ту же роль, что и интеграл энергии для консервативной системы.

$$
\text { - } 1076 \text { - }
$$




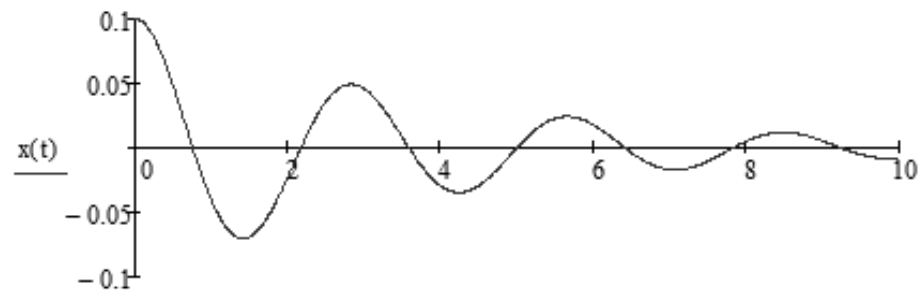

Рис. 2. Эволюция динамической системы с линейным трением во времени

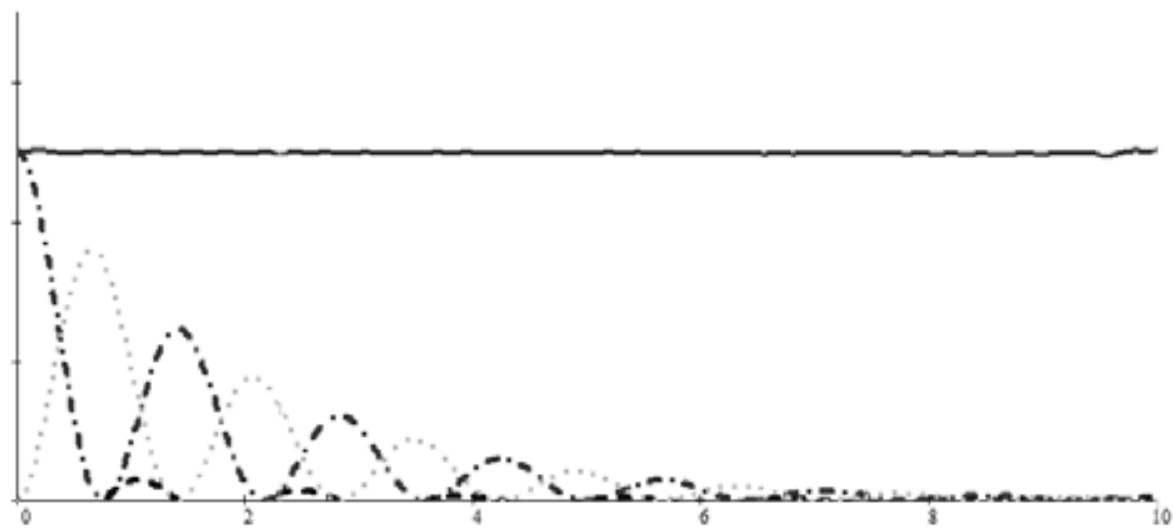

Рис. 3. Эволюция первого интеграла во времени и его компонентов: - - - потенциальная энергия, $\therefore-$ кинетическая энергия, $-2-$ работа сил трения

Первые интегралы движения имеют в механике большое значение. Даже в тех случаях, когда уравнения движения оказываются неинтегрируемыми, знание первых интегралов позволяет представить физическую картину изучаемого движения. Известные законы сохранения количества движения, кинетического момента, механической энергии системы являются первыми интегралами уравнений движения.

Исключительная общность вариационных принципов механики, возможность сравнительно простого их обобщения на многочисленные области физики, их связь с законами сохранения ставят эти принципы в центральное положение при решении многих фундаментальных проблем физики.

Как мы увидели выше, для учета диссипативных сил при исследовании динамической системы возможно определение функции Лагранжа такой системы и нахождение ее инвариантов. Все это помогает наиболее полно представить физическую картину изучаемой системы.

\section{Список литературы}

[1] Ланцош К. Вариационные принцииы механики. М., Мир, 1965. 408 c. [Lancosh K., Variation principles of mechanics. Publishing house the World, 1965. 408 p. (in Russian)]

[2] Ландау Л.Д., Лифшиц Е.М. Теоретическая физики. Т.1. Механика. 5-е изд., стереот. М., ФИЗМАЛИТ, 2004. 224 c. [Landau L.D., Lifshic E.M. Theoretical physicists. T.1. Mechanics. it is 5th edition., stereotype. M.:Fizmalit, 2004. 224 p. (in Russian)]

$$
-1077-
$$


[3] Grant R. F., George L. C. Analytical mechanics. Thomson Learning Inc. 2005.514 p.

[4] Добронравов В.В. Основы аналитической механики. М., Высш. школа, 1976. 264 с. ил. [Dobronravov V. V. Bases of analytical mechanics. M.: "Higher. school", 1976. 264 p. silt. (in Russian)] 